\title{
Bell inequalities: many questions, a few answers
}

\author{
Nicolas Gisin \\ Group of Applied Physics, University of Geneva, 1211 Geneva 4, Switzerland
}

(Dated: February 1, 2008)

\begin{abstract}
What can be more fascinating than experimental metaphysics, to quote one of Abner Shimony's enlightening expressions? Bell inequalities are at the heart of the study of nonlocality. I present a list of open questions, organised in three categories: fundamental; linked to experiments; and exploring nonlocality as a resource. New families of inequalities for binary outcomes are presented.
\end{abstract}

PACS numbers:

\section{INTRODUCTION}

This Festschrift in honor of Abner Shimony is the ideal occasion to review some of the many questions about Bell inequalities that remain open, despite more than four decades of active research and a vast number of publications on this fascinating subject. Indeed, Abner was - in modern terminology - an early adaptor of the product Bell inequality. At that time, in the 1960's and 1970's, it required quite some courage and independence of thought, two qualities characterizing Abner, to recognize the value of Bell's work on the foundations of Quantum Physics. Even in the 1980's, after Aspect's experiments, Bell inequality was still considered a dirty work. "Bohr sorted out all that years ago", was the standard answer. In those days, if you wanted your work published in PRL or similar high-standard journals you had better avoid terms like Bell inequality and (even worse) quantum nonlocality.

Starting with Artur Ekert's PRL relating Bell inequalities with quantum key distribution things have drastically changed 1]. Today it would be hard to find an issue of PRL without a mention of Bell inequality, nonlocality and - on top of it all - "the potential relevance of the presented work for quantum information processing". It is nice to see how human physicists are! And who is more human, in the most noble sense of the word, than Abner? Abner, you helped me tremendously; moreover, you did so at a time when I really needed it. Thank you Abner!

Let's return to the product Bell inequalities. Today it is fashionable, see Fig. 1, although I suspect that a large majority of physicists would still be unable to properly derive any Bell inequality. I bet that in a few decades Bell inequalities will be taught at high school, because of their mathematical simplicity, their force as an example of the scientific methodology and their huge impact on our world view. Yet, there remains a surprisingly large number of open questions, several of which are listed in section III Section IV presents a new family of Bell inequalities for an arbitrary even number of settings and binary outcomes. In appendix B an elegant Bell inequality for qubits is presented; its optimal quantum violation requires measurements of all three Pauli matrices $\sigma_{x}, \sigma_{y}$ and $\sigma_{z}$. However, let's start in section II by defining the notation.

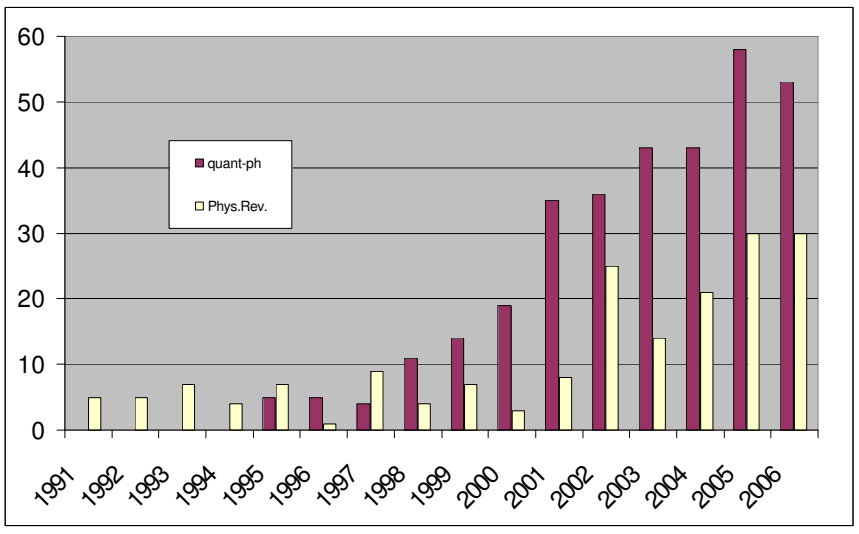

FIG. 1: (Color online) Number of occurrences of the words Bell inequality or Bell inequalities in the title or abstract of papers published during the last 16 years on the quant-ph preprint server and in Physical Review $(\mathrm{PRL}+\mathrm{PRA}+\mathrm{PRB}+\mathrm{PRC}+\mathrm{PRD}+\mathrm{PRE})$.

\section{BELL INEQUALITIES}

Bell inequalities are relations between conditional probabilities valid under the locality assumption. Hence, a priori they have nothing to do with quantum physics (and thus should not be written using quantum operators). However, it is the fact that quantum physics predicts a violation of these relations that makes them interesting. The purpose here is not to present yet another derivation of Bell inequalities, but merely to fix notation. Let $p(a, b, c, \ldots \mid x, y, z, \ldots)$ denote the conditional probability that players $A, B, C, \ldots$ produce the outcome $a, b, c, \ldots$ when they receive the input $x, y, z, \ldots$ Typically the players are physicists that perform measurements $x, y, z, \ldots$ with results $a, b, c, \ldots$ Note that $a, b, c, \ldots$ need not be numbers. We call the conditional probabilities $p(a, b, c, \ldots \mid x, y, z, \ldots)$ correlations. We assume the numbers of players, inputs and outcomes are all finite. Under the assumption of locality [i.e. there is a probability distribution $p(\lambda)$ such that $p(a, b, c, \ldots \mid x, y, z, \ldots)=$ 
$\left.\sum_{\lambda} p(\lambda) \cdot p(a \mid x, \lambda) \cdot p(b \mid y, \lambda) \cdot p(c \mid z, \lambda) \cdot \ldots\right]$ the set of all correlations is convex with finitely many vertices. Such sets are called polytopes [2]. Thus, for any given finite number of players, inputs and outcomes, the set of local correlation $p(a, b, c, \ldots \mid x, y, z, \ldots)$ is called the local polytope [2]. These polytopes are bounded by facets (hyperplanes). Each facet can be described by a linear equation: $\sum_{a, b, c, \ldots, x, y, z, \ldots} C_{a b c \ldots}^{x y z \ldots} p(a, b, c, \ldots \mid x, y, z, \ldots)=S_{l h v}$ with real coefficients $C_{a b c \ldots}^{x y z \ldots}$ and $S_{l h v}$. All local correlations lie on one side of the facet, hence they necessarily satisfy the inequality:

$$
\sum_{a, b, c, \ldots, x, y, z, \ldots} C_{a b c \ldots}^{x y z \ldots} p(a, b, c, \ldots \mid x, y, z, \ldots) \leq S_{l h v}
$$

Such inequalities are called tight Bell inequalities (for an elegant, but not tight Bell inequality, see appendix B). We say that a quantum state $\rho$ is nonlocal iff there are measurements on $\rho$ that produce a correlation that violates a Bell inequality.

The famous CHSH inequality [3] reads

$$
\begin{aligned}
& E(x=0, y=0)+E(x=0, y=1)+ \\
& E(x=1, y=0)-E(x=1, y=1) \leq 2
\end{aligned}
$$

where in our notations $E(x, y)=p(a=b \mid x, y)-$ $p(a \neq b \mid x, y)$. It is convenient to use the following selfexplanatory matrix notation:

$$
C H S H \doteq\left(\begin{array}{ll}
+1 & +1 \\
+1 & -1
\end{array}\right) \leq 2
$$

This CHSH inequality is the only tight Bell inequality for the bipartite case (i.e. two players) with binary inputs and outcomes (up to local symmetries).

Let us emphasize that the entire game consists for each player in producing, for of a given situation, a classical outcome with some probability for any possible input. In the quantum case this implies performing measurements with classical outcomes on a given quantum state $\rho$. Accordingly, the players can't combine several instances, i.e. several quantum states $\rho$, and perform quantum information processing on them, i.e. exploit coherent measurement on $\rho^{\otimes n}$ for $n \geq 2$. Note that this does not exclude the situation where the players receive a fixed number of states, like e.g. $\rho^{\otimes 3}$, but this is a different game from the one based on $\rho$. Clearly, a priori a state $\rho$ can be local, while $\rho^{\otimes n}$ is nonlocal for all $n \geq n_{\text {threshold }}>1$.

\section{OPEN QUESTIONS}

The open questions can be organized in three groups. First, the fundamental questions, most in the spirit of Bell. Next, questions more related to experiments, in the spirit of Abner's works (e.g. the famous CHSH-Bell inequality and the detection loophole). Finally, Bell-like inequalities for nonlocal resources, the most timely research on nonlocality.

Note that many open questions in quantum information theory are listen on the web page [5].

\section{A. fundamental questions}

There are infinitely many Bell inequalities. Even if one is restricted to tight Bell inequalities corresponding to facets of the polytope of local correlations, the number of Bell inequality is infinite. Restricting the given number of inputs and outcomes limits the number of Bell inequalities, but it is a computationally hard problem to list them [2].

1. Why is the CHSH inequality almost always the most efficient one to prove a quantum state to be nonlocal? Until 2004 there was no example of a quantum state not violating the CHSH inequality, but violating some other Bell inequality [4]. Still today, no natural example, i.e. a state with some natural symmetry, has been found. This leads to the concept of relevant Bell inequalities: an inequality is relevant with respect to a given set of inequalities if there is a quantum state violating it, but not violating any of the inequalities in the set.

2. Is there a finite set of inequalities such that no other inequality is relevant with respect to that set? What if one limits the dimension of the Hilbert space?

3. Find an inequality that is more efficient than the CHSH one for the Werner states [6] or prove it is impossible. In dimension two, Werner states are simply mixtures of a maximally entangled pure state $\psi$ with noise (i.e. the identity operator): $\rho_{W}=W|\psi\rangle\langle\psi|+(1-W) \mathbb{1} / 4$, where $W$ is the visibility. A local model exists for $W \lesssim 0.66$ [7], the CHSH inequality proves Werner states to be nonlocal for $W>1 / \sqrt{2}$. The region in between is unknown. The same question for the isotropic state (mixture of maximally pure state and noise) has been answered in part in [8, 9] where a generalization of the CHSH inequality to arbitrary numbers of outcomes has been shown to be more efficient. But for the isotropic states there remains also a gap in between the best known local model [10] and the proven nonlocality visibility threshold.

4. Is hidden nonlocality generic for all entangled quantum states, including mixed states? In dimension $\geq 5$, Popescu proved that the Werner states, although admitting local models, have hidden nonlocality, i.e. there are local filters such that if the Werner state passes the filters, then the resulting state violates the CHSH inequality [11], see also [12] for a simple example of hidden nonlocality. In the same vein, one should ask whether for all quantum states with hidden nonlocality there is a Bell inequality, possibly with more inputs and outcomes, that can be violated by this state? Finally, is there an example of hidden nonlocality that requires a sequence of local filters rather than a single one 
(the local model should reproduce all intermediate results)?

5. Prove some entangled quantum states to be local. This requires one to prove the existence of a local model. This has been done for Werner states (see [6] for projective measurements and [13] for general POVMs) and very recently for isotropic states [10]. A weaker form of this question asks for a proof that a state can't violate any Bell inequality with less than a given number of inputs and/or outcomes. There is only one general result to this question, see the elegant construction in [14].

6. Why are almost all known Bell inequalities for more than 2 outcomes maximally violated by states that are not maximally entangled [15]? There is quite a lot of evidence that entanglement and nonlocality are different resources [16].

7. Can all Bell inequalities with $d$ outcomes be maximally violated by a quantum state of dimension $d$ ? Or is there an example requiring states of dimension larger than the number of outcomes? In reference [17], a Bell inequality with $m$ outcomes on Alice's side and binary outcomes on Bob's side is presented. It is maximally violated by the maximally entangled state in dimension $m$.

8. Is there a local quantum state $\rho$ such that $\rho^{n}$ violates some Bell inequality? Note that if the state $\rho$ is distillable, then $\rho^{n}$, for large enough $n$, contains hidden nonlocality.

9. Find genuine n-party inequalities violated by all nparty pure entangled states. In the case of two parties, the CHSH inequality is such an example, i.e. it can be violated by any pure entangled state of whatever dimension [18, 19]. In the case of three parties there are entangled states that do not violate the MBK inequality [20, 21]. In [22, 23], a Bell inequality is presented that shows numerical evidence that all 3-party pure entangled state violate it. But the case of arbitrarily many parties is still open. Note that all n-party pure entangled states can always be projected onto a 2-party pure entangled state by projecting n- 2 parties onto appropriate local pure states [24]. This can be formulated as a tight Bell inequality where n-2 parties have only a single input. Hence, there is a set of $\left(\begin{array}{c}n \\ n-2\end{array}\right)$ inequalities that does the job. But is there a single inequality?

10. There is no known Bell inequality that requires POVMs for optimal violation on some quantum states. For binary outcomes, one can prove that POVMs are never relevant [25], but for larger a number of outcomes the question is open.
11. Almost all Bell inequalities are maximally violated by quantum states and measurements that can all be written, in an appropriate basis, using only real numbers. This is surprising since interference, a basic quantum property, "requires" complex numbers. It would be nice to find Bell inequalities suitable for distinguishing real Hilbert spaces from complex ones (i.e. an inequality that can only be violated by states and settings that require complex numbers). An example is [17].

12. Is there a bound entangled state that violates some Bell inequality? In [26] Masanes proves that no bound entangled state violates the CHSH inequality. But what about other Bell inequalities? Note that in the case of 3 players or more, it is important to distinguish different meanings of bound entanglement: bound means that the players can't distill a maximally entangled states between all of them; while totally bound means that even if some parties join into groups, they still can't distil entanglement between the groups. Dürr found a bound entangled state of 8 qubits that violates the MKB inequality [27]. However the violation is small, indicating that there is no 8-party entanglement 28]. Actually it was then demonstrated for qubits that any violation of a Bell inequality, with 2 inputs per player implies that the players, can join into groups such that the groups can distill a maximally entangled state [29, 30].

13. In the case of more than 2 parties, find inequalities testing models that assume bi-partite nonlocality but no arbitrary multi-partite nonlocality. A first example was presented already in 1987 by Svetlichny [31] and generalized in [32, 33, 34].

14. Find families of Bell inequalities valid for any number of inputs and outcomes. An example of such a family is presented in [4]. Another example is presented in this paper, see section IV] though valid only for binary outcomes and even numbers of settings. The MKB inequality 20, 21] is an example of a family of Bell inequalities with fixed numbers of inputs and outcomes, but for arbitrarily many parties. See also the recent [35].

15. Given a multi-party quantum state $\rho$, how can one know whether $\rho$ is nonlocal, i.e. whether there is a Bell inequality and measurements such that quantum physics predicts a violation of the inequality? For pairs of qubits and the CHSH inequality this problem has been solved in 1995 by the Horodecki family [36], but the general problem seems exceedingly hard. 


\section{B. Questions relevant for experiments}

The original Bell inequality [37] is, strictly speaking, not a Bell inequality according to the modern terminology that we use here. Indeed, the original inequality required, besides locality, another assumption about perfect correlations. Abner immediately recognized that this auxiliary assumption made the entire enterprize non testable and searched for an inequality involving only measurable quantities. This led him and his co-workers to find the CHSH inequality. Interestingly, the $\mathrm{CHSH}$ paper [3] already mentions the detection loophole, again underlying the importance the authors gave to the experimental issues. Concerning the detection loophole, see also 38 .

1. Find Bell inequalities easier to test experimentally with today's technology, while avoiding all known loopholes. Quantum nonlocality is so fundamental for our world view that it deserves to be tested in the most convincing way. It is thus surprising and annoying that no experiment to date has managed to close simultaneously the locality loophole (space-like separation from the choice of settings until the the classical data are secured) and the detection loophole. The latter consists in assuming that the detection efficiency is independent of the hypothetical local variables (for example, if polarization would be unknown, one would assume that all detectors are polarization insensitive, a clearly wrong assumption). Reference [39] presents a simple model reproducing all quantum correlations on maximally entangled qubits assuming detection efficiencies of $2 / 3$ (and projective measurements). Violation of the $\mathrm{CHSH}$ inequality requires detection efficiencies of at least $82.84 \%$, for maximally entangled states. There is only a single known inequality with few settings that does better, though only marginally better, $\eta_{\text {threshold }}=\sqrt{2 / 3} \approx 81.65 \%$ [40]. This inequality has 3 settings on each side and is not a facet of the polytope of local correlations. For numbers of settings larger than one hundred a better inequality has been derived from communication complexity arguments [41]. Interestingly, Philippe Eberhard noticed that partially entangled states are less sensitive to the detection loophole [42].

2. A timely variation of the previous question addresses situations where the detection efficiency differs from one side of the experiment to the other. This is natural for experiments on entanglement between quantum systems of different kinds, like e.g. an atom and a photon [43, 44, 45].

3. Find inequalities suitable for a Bell test with simple quantum-optics states and homodyne detectors. Indeed, the homodyne detection technique is well developed and always produces an outcomes. But simple cases likes, e.g. a delocalized photon in state $|0,1\rangle+|1,0\rangle$, although clearly entangled, does not violate the $\mathrm{CHSH}$ inequality with homodyne detection and a simple binarisation of the measurement results. More complicated states could violate the CHSH, but only by a tiny amount, see [46] and references therein.

4. Find inequalities for many settings. Experimentally one rarely measures precisely the four probabilities that appear in the CHSH inequality. Most of the time a series of points is measured and fitted with a sinus. Hence, an inequality for such series of points could be more appropriate. Examples are given in [47, 48] and in section IV.

\section{Bell-like inequalities for nonlocal resources}

This subsection presents recently opened questions and moves away from the traditional work on Bell inequalities. It starts by admitting quantum nonlocality and aims at better quantifying it and at understanding it as a new kind of resource. These questions investigate nonlocal but non-signaling correlations [49]. Recall that a correlation $p(a, b, c, \ldots \mid x, y, z, \ldots)$ is nonsignaling iff all the marginals are independent of the other players's inputs: $\sum_{b, c, \ldots} p(a, b, c, \ldots \mid x, y, z, \ldots)=p(a \mid x)$, $\sum_{a, c, d, \ldots} p(a, b, c, \ldots \mid x, y, z, \ldots)=p(b, \mid y)$, etc.

Bell inequalities are tests for correlations that can be simulated using only local resources and shared randomness (a modern terminology for the obsolete local hidden variables). This view raises the question of correlations that can be simulated using, in addition to shared randomness, some finite amount of some given nonlocal resource. For example, it is known that any pair of projective (Von Neumann) measurements on any maximally entangled state of two 2-level quantum systems can be simulated using only shared randomness and a single PRbox (a sort of unit of nonlocality) [50, 51, 52, 53]. Hence, it is interesting to characterize all correlations that can't be simulated using shared randomness and one PR-box. Surprisingly, some correlations resulting from quantum measurements on partially entangled 2-level systems are of that kind.

1. Is there a Bell-like inequality valid for all correlations simulable with a single bit of communication and violated by some partially entangled 2-qubit states? Actually, the entire field of research considered in this subsection started with a paper presenting Bell-like inequalities valid for 1 bit of communication 54]. However, the presented inequalities can't be violated by any 2 -qubit states. We know that maximally entangled 2-qubit states can be simulated with a single bit of communication; thus such states don't violate any of the considered Bell-like inequalities. However, the question remains open for partially entangled states. 
2. Are all partially entangled qubit pairs not simulable by a single PR-box? A few Bell-like inequalities satisfied by all correlations simulable by a single PR-box and shared randomness are known [55, 56]. From these one knows that very poorly entangled states can't be simulated with one PR-box, but the case of high-but-not-maximally entangled states is open.

3. Find inequalities satisfied by all correlation that can be simulated by two PR-boxes. Two bits of communication suffice to simulate any two qubit state. Is the same true for two PR-boxes?

4. Find any non-signaling box [49] with finitely many inputs and outcomes with which one can simulate partially entangled states.

5. Find the Quantum-Bell inequalities that bound the correlations achievable with quantum measurements and states? An example is the Tsirelson bound [57] stating that quantum correlations can't violate the $\mathrm{CHSH}$ inequality by more than the well known factor $2 \sqrt{2}$, see also [58].

6. Can a secret key be distilled out of any nonlocal correlation, (secret against any non-signaling adversary performing arbitrary individual attacks) $[59,60,61]$ ? This question may appear to move away from Bell questions, but it concerns the power of the nonlocal resources as witnessed by Bell inequalities. It also addresses the question of the existence of bound information [62, 63], a classical analog to bound entanglement.

\section{THE AS-BELL INEQUALITY FAMILY}

I know of only a single family of bipartite Bell inequalities valid for any number of inputs and outcomes [4]. In this section I briefly present a new family of bipartite Bell inequalities for any even number of inputs and binary outcomes. I found this family by looking for correlation Bell inequalities with a few inputs and binary outcomes. Recall that a correlation inequality involves only expectation values: $E(x, y)=p(a=b \mid x, y)-p(a \neq b \mid x, y)$. For binary inputs, the CHSH is the only inequality. For ternary inputs, there is no new correlation inequality [4, 64]. For 4 inputs on each side, I searched numerically all possibilities assuming small integer coefficient. I found only two new inequalities (the coefficient in the matrix indicate the coefficients of the corresponding expectation values):

$$
A S_{4} \doteq\left(\begin{array}{cccc}
+1 & +1 & +1 & +1 \\
+1 & +1 & +1 & -1 \\
+1 & +1 & -2 & 0 \\
+1 & -1 & 0 & 0
\end{array}\right) \leq 6
$$

$$
D_{4} \doteq\left(\begin{array}{llll}
+2 & +1 & +1 & +2 \\
+1 & +1 & +2 & -2 \\
+1 & +2 & -2 & -1 \\
+2 & -2 & -1 & -1
\end{array}\right) \leq 10
$$

Avis and co-workers demonstrated that these are indeed the only correlation inequalities for 4 inputs 65]. Inspired by inequality $A S_{4}$, it is not difficult to guess the form of the next inequalities:

$$
\begin{gathered}
A S_{6} \doteq\left(\begin{array}{cccccc}
+1 & +1 & +1 & +1 & +1 & +1 \\
+1 & +1 & +1 & +1 & +1 & -1 \\
+1 & +1 & +1 & +1 & -2 & 0 \\
+1 & +1 & +1 & -3 & 0 & 0 \\
+1 & +1 & -2 & 0 & 0 & 0 \\
+1 & -1 & 0 & 0 & 0 & 0
\end{array}\right) \leq 12 \quad(6) \\
A S_{8} \doteq\left(\begin{array}{cccccccc}
+1 & +1 & +1 & +1 & +1 & +1 & +1 & +1 \\
+1 & +1 & +1 & +1 & +1 & +1 & +1 & -1 \\
+1 & +1 & +1 & +1 & +1 & +1 & -2 & 0 \\
+1 & +1 & +1 & +1 & +1 & -3 & 0 & 0 \\
+1 & +1 & +1 & +1 & -4 & 0 & 0 & 0 \\
+1 & +1 & +1 & -3 & 0 & 0 & 0 & 0 \\
+1 & +1 & -2 & 0 & 0 & 0 & 0 & 0 \\
+1 & -1 & 0 & 0 & 0 & 0 & 0 & 0
\end{array}\right) \leq 20
\end{gathered}
$$

The generalization to arbitrary even number of inputs is straightforward. Note that $A S_{2}$ is nothing but the CHSH inequality. Numerically, these $A S_{n}$ inequalities are tight and maximally violated by maximally entangled qubit states for visibilities larger than $V_{n}$, with $V_{2}=1 / \sqrt{2} \approx 0.7071, V_{4} \approx 0.7348, V_{10} \approx 0.7469$, $V_{32} \approx 0.7497, V_{50} \approx 0.7499$. Apparently $V_{\infty} \approx 0.75$; this contrasts with the $I_{n n 22}$ family presented in [4] where for binary outcomes and large numbers of inputs the threshold visibility appears to tends to 1 . All settings can be chosen to lie on a grand circle of the Poincaré sphere.

\section{CONCLUSION}

We are lucky to live at the time where physics discovers and explores the nonlocal characteristics of Nature. Contrary to the nonlocality of Newtonian gravitation, quantum nonlocality is with us for ever [66, 67]. Future historians of Science will describe our epoch as that of the great discovery of nonlocality. The name of Abner Shimony will forever be associated with this fascinating epoch.

The choice of questions listed in this contribution to Abner's Festschrift is necessarily somewhat subjective. Others may like to add their favorite ones or to formulate the questions differently. Important is the fact that there are many interesting open questions of very different kinds. The basic maths is simple, but a deeper understanding requires concepts ranging from combinatorial and complexity theories to algebra and geometry in high dimensions. Hence, it is likely that most of the 
listed problems are hard. But their solutions, even partial solutions, will be valuable contributions to one of the most fascinating research fields of the 21st century.

\section{Acknowledgment}

This work has been supported by the EC under project QAP (contract n. IST-015848) and by the Swiss NCCR Quantum Photonics. Thanks are due to Rob Thew, Toni Acin, André Méthot, Sandu Popescu and Valerio Sacarani for their comments on previous versions of this paper.

\section{Appendix A: Some diagonal Bell inequalities}

Correlation Bell inequalities of a form similar to D4 (eq. 5) can easily be found numerically. For 5 inputs on each side there seems to exist only two such inequalities (at least I found only two). They are entirely defined by their first line and the permutation rule as in (5) (from one line to the next: shift each entry to the left, the entry that falls out is re-introduced on the right hand side with the opposite sign):

$$
\begin{aligned}
& D 5_{1} \doteq(11011) \leq 8 \\
& D 5_{2} \doteq(32113) \leq 20
\end{aligned}
$$

For 6 inputs I found:

$$
\begin{aligned}
& D 6_{1} \doteq(101011) \leq 10 \\
& D 6_{2} \doteq(311124) \leq 28 \\
& D 6_{3} \doteq(422125) \leq 36 \\
& D 6_{4} \doteq(422136) \leq 42
\end{aligned}
$$

For more inputs, the numbers of such D-inequalities seems to grow rapidly.

\section{Appendix B: An elegant Bell inequalities}

In ref. [17] Helle Bechmann-Pasquinucci and myself presented a Bell inequality tailored for quantum cryptography in high dimension Hilbert spaces. Since this inequality seems to have a few original features, like being optimally violated by states and quantum measurements requiring complex numbers and Hilbert spaces of dimension larger than the number of outcomes on Bob's side (but equal to the number of outcomes on Alice's side), I recall it in this appendix with the notations used throughout this contribution. Moreover, this new way of looking at this inequality underlines its similarity with communication complexity [68].

In this game, Alice receives as input a number $x \in$ $\{0,1, \ldots, n-1\}$, while Bob's input consists of $n$ numbers $y_{0}, y_{1}, \ldots, y_{n-1}$ with each $y_{j} \in\{0,1, \ldots, m-1\}$. Basically, the goal is that Alice outputs $a=y_{x}$. As such this would be merely an example of a communication complexity game. But in our game, Bob can use a joker and refuse that this instance of the game counts. Accordingly, Bob's outcome is binary. Whenever $b=0$, the score is null, whatever Alice's outcome. Whenever $b=1$ the score is +1 if $a=y_{x}$ and -1 if $a \neq y_{x}$. Explicitly, the Bell inequality reads:

$$
\begin{gathered}
S H B= \\
\sum_{\substack{x=0 \ldots n-1 \\
y_{0} \ldots y_{n-1}=0 \ldots m-1}} \begin{array}{r}
\left(p\left(a=y_{x}, b=1 \mid x, y_{0}, \ldots, y_{n-1}\right)\right. \\
\left.-p\left(a \neq y_{x}, b=1 \mid x, y_{0}, \ldots, y_{n-1}\right)\right)
\end{array}
\end{gathered}
$$

$$
\leq S_{\text {local }}
$$

The optimal local strategy consists of Alice and Bob agreeing in advance on a sequence $y_{0}^{g}, \ldots, y_{n-1}^{g}$ and Alice producing $a=y_{x}^{g}$ while Bob accepts the game only for the inputs $y_{0}, \ldots, y_{n-1}$ for which the averaged score is positive:

$$
S_{\text {local }}=\sum_{r=0}^{\left[\frac{n-1}{2}\right]}(n-2 r)\left(\begin{array}{l}
n \\
r
\end{array}\right)
$$

Let us concentrate on the case $n=2$ for which $S_{\text {local }}=2$. The optimal quantum strategy requires Alice and Bob to share a maximally entangled state of dimension $m$. Alice measures her quantum system in one out of two mutually conjugated bases, depending on her input $x=0$ or $x=1$. Bob receives two symbols as input, $y_{0}$ and $y_{1}$, corresponding to two quantum states, one in each of the two bases. He applies to his quantum system a measurement described by the projector onto the state which lies precisely in between the two states that correspond to $y_{0}$ and $y_{1}$ (since the two states belong to two mutually conjugated bases, such an intermediate state is always uniquely define. Take for instance the eigenstate with maximal eigenvalue of the density matrix obtained by a $50-50 \%$ mixture of the two states). If Bob's projection is successful, this projects Alice's state onto the state that maximizes her chance of finding the correct outcome. In such a case Bob outputs $b=1$. In the alternative case, i.e. failure of his projection measurement, he outputs $b=0$; that is, Bob's outcome is his measurement result. With this quantum strategy, Alice and Bob beat the optimal local strategy by a factor $\sqrt{m}$ :

$$
S_{\text {quantum }}=2 \sqrt{m}>S_{\text {local }}=2
$$

Note that for $m=2$, this reduces to the well studied CHSH inequality. Indeed, although in this case Bob has formally four possible inputs, the corresponding four projectors form two bases. Explicitly, Alice measures one of the two operators $\sigma_{x}$ or $\sigma_{z}$, depending on her input, and Bob measures in the intermediate bases $\sigma_{+45^{\circ}}$ (for his inputs 0,0 and 1,1$)$ or $\sigma_{-45^{\circ}}$ (for inputs 0,1 and 1,0 ). 




FIG. 2: (Color online) Measurement settings represented on the Poincaré sphere for the elegant inequality $S_{3 \times 4}$ defined by eq. (17). Alice's 3 settings are represented by 3 mutually orthogonal vectors, and Bob's 4 settings by the vertices of the tetrahedron.

For $n=2$ and $m=3$ the quantum optimum of $2 \sqrt{3} \approx$ 3.464 is reached by the strategy summaried above and presented in [17]. Numerical evidence suggests that if one restricts oneself to settings that can be expressed using only real numbers, the maximum is slightly lower: $10 / 3 \approx 3.333[17$. Moreover, this maximum is reached for a non-maximally entangled state. But it is unknown whether a higher score can be achieved using only real numbers in larger Hilbert spaces.

The case $n=3, m=2$ appears also to be interesting. Indeed, the quantum maximum is $4 \sqrt{3} \approx 6.928$, while the maximum using only real numbers is reached by the singlet state at $2+2 \sqrt{5} \approx 6.472$. This might open the possibility to test correlations requiring complex Hilbert spaces (however, here again it remains to test the inequality in higher dimensions).

Note that this inequality $n=3, m=2$ can also be written as a correlation inequality. Indeed, Bob's $m^{n}=8$ inputs can be grouped into 4 projective measurements. In this form, this inequality reads:

$$
S_{3 \times 4} \doteq\left(\begin{array}{ccc}
+1 & +1 & +1 \\
+1 & -1 & -1 \\
-1 & +1 & -1 \\
-1 & -1 & +1
\end{array}\right) \leq 6
$$

Another elegant feature of this case $n=3, m=2$ is seen when the optimal settings are represented on the Poincaré sphere: for Alice the three vectors are mutually orthogonal, while Bob's four vectors are on the vertices of the tetrahedron, see Fig. 2.

To conclude, let us note that most inequalities presented in this appendix, in particular the elegant $S_{3 \times 4}$, are not facets of the local polytope. This indicates that the geometry of the local polytope doesn't match the symmetries of elegant quantum states and measurements. In the case of 3 and 4 inputs on Alice and Bob's side, respectively, all facets are known [4], hence one shouldn't be surprised that the new inequality $S_{3 \times 4}$ is not a facet.
[1] A.K. Ekert, Quantum cryptography based on Bell's theorem. Phys. Rev. Lett. 67, 661-663 (1991).

[2] I. Pitowski, Quantum Probability - Quantum Logic, Springer-Verlag 1989.

[3] J.F. Clauser, M.A. Horne, A. Shimony, and R.A. Holt, Proposed Experiment to Test Local Hidden-Variable Theories. Phys. Rev. Lett. 23, 880 (1969).

[4] D. Collins, and N. Gisin, A relevant two qubit Bell inequality inequivalent to the CHSH inequality. J. Phys. A: Math. Gen. 37, 1775 (2004).

[5] http : //www.imaph.tu - bs.de/qi/problems

[6] R.F. Werner, Phys. Rev. A 40, 4277 (1989).

[7] A. Acin, N. Gisin and B. Toner, Phys. Rev. A 73, 062105 (2006).

[8] D. Kaszlikowski et al., Phys. Rev. Lett. 85, 4418 (2000).

[9] D. Collins, N. Gisin, N. Linden, S. Popescu and S. Massar, Phys. Rev. Lett. 88, 040404 (2002).

[10] M.L. Almeida et al, quant-ph/0703018.

[11] S. Popescu, Phys. Rev. Lett. 74, 2619-2622 (1995).

[12] N. Gisin, Phys. Lett. A 210, 151-156 (1996); ibid. 224, 317-318 (1997).

[13] J. Barrett, Phys. Rev. A bf65, 042302 (2002).

[14] B.M. Therhal, A. Doherty and D. Schwab, Phys. Rev. Lett. 90, 157903 (2003).
[15] A. Acin, T. Durt, N. Gisin and J. I. Latorre, Phys. Rev. A 65, 052325 (2002).

[16] A.A. Méthot and V. Scarani, Quant. Inf. Comput. 7, 157 (2007).

[17] H. Bechmann-Pasquinucci and N. Gisin, Phys. Rev. A 67, 062310, 2003. See also appendix B.

[18] N. Gisin, Phys. Lett. A 154, 201-202 (1991).

[19] N. Gisin and A. Peres, Phys. Lett. A 162, 15-17 (1992).

[20] N.D. Mermin, Phys. Rev. Lett. 65, 1838 (1990).

[21] A.V. Belinskii and D.N. Klyshko, Phys. Usp. 36, 653 (1993).

[22] A. Acin et al., Phys. Rev. Lett. 92, 250404 (2004).

[23] J.-L. Chen, Phys. Rev. Lett. 93, 140407 (2004).

[24] S. Popescu and D. Rohrlich, Phys. Lett. A 166, 293-297 (1992).

[25] R. Cleve, P. Hoyer, B. Toner and J. Watrous, quant$\mathrm{ph} / 0404076$.

[26] L. Masanes, Phys. Rev. Lett. 97, 050503 (2006).

[27] W. Dür, Phys. Rev. Lett. 87, 230402 (2001).

[28] N. Gisin and H. Bechmann.Pasquinucci, Phys. Lett. A 246, 1-6 (1998).

[29] A. Acin, Phys. Rev. Lett. 88, 027901 (2001).

[30] A. Acin, V. Scarani and M. Wolf, Phys. Rev. A 66, 04323 (2002). 
[31] G. Svetlichny, Phys. Rev. D 35, 3066 (1987).

[32] D. Collins et al., Phys. Rev. Lett. 88, 170405 (2002).

[33] M. Seevinck and G. Svetlichny, Phys. Rev. Lett. 89, 060401 (2002).

[34] J.L. Cereceda, Phys. Rev. A 66, 024102 (2002).

[35] K. Nagata, W. Laskowski and T. Paterek, Phys. Rev. A 74, 062109 (2006).

[36] M. Horodecki, P. Horodecki and R. Horodecki, Phys. Lett. A 200, 340 (1995).

[37] J.S Bell, Physics 1, 195-200 (1964); reprinted in: J.S. Bell, Speakable and Unspeakable in Quantum Mechanics: Collected papers on quantum philosophy, (Cambridge University Press, Cambridge, 1987, revised edition 2004).

[38] Ph. Pearle, Phys.Rev. D 2, 1418 (1970).

[39] B. Gisin and N. Gisin, Phys. Lett. A 260, 323-327 (1999).

[40] S. Massar, S. Pironio, J. Roland and B. Gisin, Phys. Rev. A 66, 052112 (2002).

[41] H. Buhrman, P. Hoyer, S. Massar and H. Roehrig, Phys. Rev. Lett. 91, 047903 (2003).

[42] Ph. Eberhard, Phys. Rev. A 47, R747 (1993).

[43] J. Volz et al., Phys. Rev. Lett. 96, 030404 (2006).

[44] A. Cabello and J.-A. Larsson, quant-ph/0701191, , Phys. Rev. Lett. in press (2007).

[45] N. Brunner, N. Gisin, V. Scarani and Ch. Simon, quantph/0702130, Phys. Rev. Lett. in press (2007).

[46] R. Garca-Patrón, J. Fiurácek, N. J. Cerf, J. Wenger, R. Tualle-Brouri and Ph. Grangier, Phys. Rev. Lett. 93, 130409 (2004).

[47] M. Zukowski, Phys. Lett. A 177, 290 (1993). See also quant-ph/9908009.

[48] N. Gisin, Phys. Lett. A 260, 1-3 (1999).

[49] J. Barrett et al., Phys. Rev. A 71, 022101 (2005).

[50] S. Popescu and D. Rohrlich, Foundations of Physics 24, 379-385, (1994).

[51] N. Cerf, N. Gisin and S. Massar, Phys. Rev. Lett. 94, 220403 (2005).
[52] J. Barrett and S. Pironio, Phys. Rev. Lett. 95, 140401 (2005).

[53] F. Dupuis et al., quant-ph/0701142.

[54] D. Bacon and B. Toner, Phys. Rev. Lett. 90, 157904 (2003).

[55] N. Brunner, N. Gisin and V. Scarani, New J. Phys. 7, 1-14 (2005).

[56] N. Brunner, V. Scarani and N. Gisin, J. Math. Phys. 47, 112101 (2006).

[57] B.S. Tsirelson, Lett. Math. Phys. 4, 83, 1980.

[58] M. Navascues, S. Pironio and A. Acin, quant$\mathrm{ph} / 0607119$.

[59] J. Barrett, L. Hardy and A. Kent, Phys. Rev. Lett. 95, 010503 (2005).

[60] V. Scarani, N. Gisin, N. Brunner, L. Masanes, S. Pironio and A. Acin, Phys. Rev. A 74, 042339 (2006).

[61] A. Acin, N. Gisin and Lluis Masanes, Phys. Rev. Lett. 97, 120405 (2006).

[62] N. Gisin and S. Wolf, Phys. Rev. Lett. 83, 4200-4203 (1999).

[63] N. Gisin, R. Renner and S. Wolf, Algorithmica 34, 389412 (2002).

[64] C. Sliwa, Phys. Lett. A 317, 165-168 (2003).

[65] D. Avis, H. Imai and T. Ito, J. Phys. A 39, 11283-11299 (2006).

[66] Isaac Newton, Papers \& Letters on Natural Philosophy and related documents, page 302, Edited, with a general introduction, by Bernard Cohen, assisted by Robert E. Schofield Harvard University Press, Cambridge, Massachusetts, 1958

[67] N. Gisin, Can relativity be considered complete ? From Newtonian nonlocality to quantum nonlocality and beyond, quant-ph/0512168.

[68] Brassard, G., $Q$ communication complexity, quant$\mathrm{ph} / 0101005$. 